\title{
Mites Associated with Poultry and Rabbits
}

\author{
A. M. Metwally*; Hala M. Gamal El-Din **; A. A. Abdallah*and Amany A. El-Sadawy** \\ *Faculty of Agriculture, Al-Azhar University, Cairo, Egypt. \\ ${ }^{* *}$ Faculty of Home Economics, Al-Azhar University, Tanta, Egypt.
}

\begin{abstract}
Sixty nine mite species belonging to 2 orders, 5 suborders and 34 families were collected from poultry and rabbits at different location of Egypt during two years (2013-2014). Suborder Gamasida included 30 species of 11 families; suborder Actinedida 17 species of 13 families; suborder Acaridida 17 species of 6 families; suborder Oribatida 2 species of 2 families; and suborder Ixodida one species of one family.
\end{abstract}

Key words: Incidence; Mites; Poultry, Rabbits.

\section{INTRODUCTION}

Mites comprise a large economic group of Arthropoda found in almost every habitat suitable to animal life. Careful examination of the nearest handful of litter under domestic or wild agricultural animals will surely uncover several species. Some are living on minute particles of food. Activities of a few species are well known, people acquainted with their ravage can rarely recognize the mites if they see them. Whoever. However, the small size of mites is responsible for the lack of information available on them.

Mites are so vary in their feeding habits; some are phytophagous, pests of stored products, predators, animal parasites and free livings. However, mites significantly affect the agriculture, food, health and physical comfort of our society.

Rabbit production is one of the fastest growing livestock enterprises globally.

Abo-Taka (1996) examined poultry houses at six localities of Egypt, and determined the prevalence of different mite species inhabiting chicken, ducks and pigeons. Astigmatid mites had in the highest proportion while mesostigmatids had the lowest.

The red mite, Dermanyssus gallinae is an example of poultry parasite that also being injurious to man .It was recorded on domestic fowls, pigeons and other birds (Abo-Taka \&Allam, 1997).

Mahgoob et al. (2006) collected different samples from litter, poultry feed and feather from chickens, ducks, pekeeny ducks and geese and hair from rabbits and feathers from quails, rozella birdsand zebra, birds (as wild animals which were in captivity) and found twenty-seven families of the suborders; Gamasida, Actinedida, Acaridida and Oribatida plus hypopal stage of the family Acaridae.
Therefore, this study dealt with surveying mites associated with poultry and rabbit farm.

\section{MATERIALS AND METHODS}

\section{Mites' collection:}

Samples were collected monthly from poultry and rabbit litter and feaces during two successive years (2012-2013) and (2013-2014) in plastic bags sent to the laboratory for mites separation. Collected mites were isolated in Nesbitt's solution for clearing then mounted in Hoyer's Medium on glass slides for examining. Mites were identified and kept in the collection at the Department of Agricultural Zoology and Nematology, Faculty of Agriculture Al-Azhar University.

\section{Mite's identification:}

Mite identification was conducted according Griffiths, 1960; Hughes, 1961 \&1976; Lindquist and Evans, 1965; Summers and Price, 1970; Karg, 1971; Zaher et al, 1986 and Krantz \& Walter, 2009.

\section{RESULTS AND DISCUSSION}

\section{Incidence of mite families:}

Mites associated with poultry (domestics birds and rabbits) were recorded during the two successive years (2012-2014).

\section{Order Parasitiformes:}

This order was represented by the two suborders: Gamasida which included 11 families Ascidae, Ameroseiidae, Rhodacaridae, Macrochelidae, Digamaseilidae, Ologamasidae, Laelapidae, Macronyssidae, Dermanysidae, Uropodidae and Parasitidae; and thirty species and the suborder Ixodida which included one species belonging to the family Argasidae (Table 1).

\section{Order Acariformes:}

This order was represented by three suborders: Actinedida which included 12 families Cheyletidae, 
Tydeidae, Tarsonemidae, Eupodidae, Rhagidiidae, Bdellidae, Cunaxidae, Acarophenacidae, Pygmephoroidae, Sitroptidae, Scutacaridae and Psorpptidae; Suborder Acaridida which included
6 families Analgidae, Pyroglyphidae, Acaridae, Chrotoglyphidae, Suidasiidae and Glycyphagidae; and Suborder Oribatida which included 2 families Oribatulidae and Oppidae (Table 2).

Table (1): Incidence of mite species belonging to order Parasetifomes associated with poultry and rabbits in

Gharbia, Kafr El-Shiekh and Cairo governorates for two successive years (2012- 2014)

\begin{tabular}{|c|c|c|c|c|}
\hline \multicolumn{5}{|c|}{$\begin{array}{l}\text { Order: Parasetiformes } \\
\text { Suborder: Gamasida }\end{array}$} \\
\hline Family & Species & Habitat & Abundance & Locality \\
\hline \multirow{5}{*}{ Ascidae (5) } & Proctolaelaps pygmaeus Muller & \multirow[b]{2}{*}{ Chicken-Ducks-Geese } & \multirow[b]{2}{*}{++} & \multirow[b]{2}{*}{ Qotor-Sakha } \\
\hline & $\begin{array}{l}\text { Proctolaelaps bickley } \\
\text { (Lindiqucot\&Huntaer) }\end{array}$ & & & \\
\hline & Protogamasellus minutus Nasr & $\begin{array}{c}\text { Pigeon-Ducks-Geese -Turkey } \\
\text { Hen }\end{array}$ & ++ & Nasr city-Sakha \\
\hline & Protogamasellus denticus Nasr & \multirow{2}{*}{ Quails-pigeon } & \multirow{2}{*}{++} & \multirow{2}{*}{ Nasr city } \\
\hline & Blattisocius tarsalis (Berlese) & & & \\
\hline \multirow{2}{*}{ Ameroseiidae (2) } & Kleemania plumosus (Oudemans) & \multirow{2}{*}{$\begin{array}{l}\text { Chicken-ducks-pekeenyducks-- } \\
\text { Geese }\end{array}$} & \multirow{2}{*}{++} & \multirow{2}{*}{$\begin{array}{l}\text { Nasr city-Qotor- } \\
\text { Sakha } \\
\end{array}$} \\
\hline & Kleemania plumigera (Berlese) & & & \\
\hline \multirow{3}{*}{ Macroohelidae (3) } & Macrocheles merdarius (Berlese) & Ducks- Turkey Hen-Rabbits & ++ & Qotor-Tanta \\
\hline & Macrocheles matrius (Hull) & Pekeeny-Ducks-Rabbits & ++ & Qotor \\
\hline & Macrocheles pisentii & Ducks-Turkey Hen-Rabbits & ++ & Nasr city-Sakha \\
\hline \multirow[b]{2}{*}{ Rhodacaridae (2) } & Rhohacarus roseus (Oudemans) & \multirow[b]{2}{*}{ Ducks-Pigeon-Chicken } & \multirow[b]{2}{*}{++} & \multirow[b]{2}{*}{ Qotor-Sakha } \\
\hline & $\begin{array}{l}\text { Rhohacarus subterraneus } \\
\text { (Willnan) }\end{array}$ & & & \\
\hline $\begin{array}{l}\text { Digamasellidae(1 } \\
\text { ) }\end{array}$ & $\begin{array}{l}\text { Dendrolaelaps aegypticus } \\
\text { Metwally\&Mersal }\end{array}$ & Turkey Hen & + & \\
\hline Ologamasidae (1) & Gamasiphis parpulchellus Berlese & Turkey Hen-Rabbits & + & Nasr city \\
\hline \multirow[b]{2}{*}{ Dermanyssidae (2) } & Dermanyssus gallinae (De Geer) & \multirow[b]{2}{*}{ Pigeon -Quails } & \multirow[b]{2}{*}{++} & \multirow[b]{2}{*}{ Tanta } \\
\hline & $\begin{array}{l}\text { Dermanyssus chellidonis } \\
\text { (Oudemans) }\end{array}$ & & & \\
\hline \multirow{5}{*}{ Macronyssidae (5) } & Steatonyssus occidentalis (Ewing) & \multirow{3}{*}{ Ducks-Pigeon-Quails } & \multirow{3}{*}{+++} & \multirow{3}{*}{ Sakha-Tanta } \\
\hline & $\begin{array}{l}\text { Macronyssus longisetosus } \\
\text { (Fuymar) }\end{array}$ & & & \\
\hline & Macronyssus japonica Rodovesky & & & \\
\hline & Ornithonyssus bacoti (Hirst) & \multirow[b]{4}{*}{ Ducks-Turkey Hen-Rabbits } & \multirow{2}{*}{ H } & \multirow{2}{*}{$\begin{array}{l}\text { Nasr city } \\
\text { TantaQotor }\end{array}$} \\
\hline & $\begin{array}{l}\text { Ornithonyssus pipstrelli } \\
\text { (Oudemans) }\end{array}$ & & & \\
\hline \multirow{5}{*}{ Laelapidae (5) } & Androlaelaps casalis (Berlese) & & & \\
\hline & $\begin{array}{l}\text { Androlaelaps kefli } \\
\text { (Metwally\&Ibrahim) }\end{array}$ & & $+1+$ & Qotor -Sakha \\
\hline & $\begin{array}{l}\text { Hypoaspis wahabi } \\
\text { (Metwally\&Ibrahim) }\end{array}$ & Quails-Pigeons & $+1+$ & inta-Qotor-Sakha \\
\hline & Hypoaspis oblanga (Halbert) & & & \\
\hline & Laelapis hilaris (Koch) & Ducks-PeckenyDucks & ++ & Tanta-Qotor \\
\hline & Uroobovella margineda (Berlese) & & & \\
\hline Uropodidae (3) & $\begin{array}{l}\text { Trichouropoda potaviana } \\
\text { (Canestrini) }\end{array}$ & Ducks-Turkey Hen-Rabbits & ++ & Qotor-Sakha \\
\hline & Rhychopolus rhychophori (Oliver) & & & \\
\hline Parasitidae (1) & $\begin{array}{l}\text { Parasitus consanguineus } \\
\text { Oudemans\&Voigts }\end{array}$ & Ducks-Turkey Hen-Rabbits & ++ & Qotor-Sakha \\
\hline & Subor & ler: Ixodida & & \\
\hline Argasidae (1) & Ornithorous erraticus & Pigeon Chickens - Quails & ++ & city- Sakha-Qotor \\
\hline
\end{tabular}


Table (2): Incidence of mite species belonging to order Acarifomes associated with poultry and rabbits in Gharbia, Kafr El-Shiekh and Cairo governorates for two successive years (2012- 2014)

\begin{tabular}{|c|c|c|c|c|}
\hline \multicolumn{5}{|c|}{ Suborder: Actinedida } \\
\hline Family & Species & Habitat & Abundance & Locality \\
\hline \multirow{4}{*}{ Cheyletidae (5) } & Acaropsella notchi omaa\&Hassan & \multirow{2}{*}{$\begin{array}{c}\text { Chicken-Ducks-Peckeny-Ducks- } \\
\text { Pigeon }\end{array}$} & \multirow{2}{*}{++} & \multirow{2}{*}{ Tanta } \\
\hline & Eucheyletia sp. & & & \\
\hline & Cheyletus malaccensis Oudemans & \multirow{2}{*}{$\begin{array}{l}\text { Poultry food-Chicken-Ducks- } \\
\text { Turkey-Hen-Pigeon-Quails }\end{array}$} & \multirow{2}{*}{++} & \multirow{2}{*}{$\begin{array}{l}\text { Nasr city -Qotor- } \\
\text { Sakha }\end{array}$} \\
\hline & Cheyletus eruditus (Schrank) & & & \\
\hline Tydeidae (1) & Parraloryia sp. & Litter of poultry food & ++ & Tanta-Sakha \\
\hline Tarsonemidae(1) & Tarsonemus sp. & Litter of all poultry & ++++ & Tanta-Nasr city \\
\hline Eupodidae (1) & Eupodes momeni Abou-Awad & Pigeon -Quails & ++ & $\begin{array}{l}\text { Qotor - Tanta- Nasr } \\
\text { city }\end{array}$ \\
\hline Rhagidiidae (1) & $\begin{array}{c}\text { Neothoria niloticus Abou- } \\
\text { Awad\&El-Bagoury }\end{array}$ & Pekeeny-Ducks-Turkey-Hen & ++ & $\begin{array}{l}\text { Qotor-Sakha-Nasr } \\
\text { city }\end{array}$ \\
\hline Bdellidae (1) & Spinibdella bifurcate Atyeo & Ducks-Rrabbits & ++ & $\begin{array}{l}\text { Tanta-Sakha-Nasr } \\
\text { city }\end{array}$ \\
\hline Cuaxidae (1) & Puleaus glebulentus Den Heyer & Turkey Hen-Rabbits & ++ & Qotor-Sakha-Tanta \\
\hline Acarophenacidae (1) & Acarophinax sp. & Ducks-Turkey Hen-Rabbits & ++ & Tanta -Sakha \\
\hline Pygmephoroidae (1) & Trochometridium sp. & Ducks-Turkey Hen-Rabbits & +++ & Tanta \\
\hline Sitroptidae (1) & $\begin{array}{c}\text { Sitroptes serrateseta } \\
\text { Soliman\&Kandeel }\end{array}$ & Pigeon -Quails & + & Nasr city - Qotor \\
\hline Scutacaridae (1) & Scutacarus sp. & Chicken-Pigeon-Quails & ++ & Qotor-Tanta \\
\hline \multirow{2}{*}{ Psorpptidae (2) } & Psorpties communis Ovis & Rabbits & ++++ & Tanta \\
\hline & Chorioptes sp. & Rabbits & ++++ & Tanta \\
\hline \multicolumn{5}{|c|}{ Suborder: Acaridida } \\
\hline \multirow{2}{*}{ Analgidae (2) } & Trouessart sp. & & + & \\
\hline & Analges corrinus (Megnin) & Chicken-Geese-Pigeon-Quails & + & Nasr city \\
\hline \multirow{2}{*}{ Pyroglyphidae (2) } & Dermatophagides farina (Hughes) & Rabbits & ++ & Tanta- Sakha \\
\hline & Pyroglyphus sp. & Rabbits & ++ & Tanta-Sakha \\
\hline \multirow{8}{*}{ Acaridae (9) } & Tyrophagous putrescentiae (Shank) & Chicken-Ducks-Geese-Pigeon & +++ & Tanta \\
\hline & Tyrophagous palmarum Oudemans & Poultry food & +++ & $\begin{array}{l}\text { Tanta-Qotor-Nasr } \\
\text { city }\end{array}$ \\
\hline & Risoglyphus calleae Oudemans & Ducks-Geese-Turkey Hen & ++++ & $\begin{array}{l}\text { Tanta-Qotor-Nasr } \\
\text { city } \\
\end{array}$ \\
\hline & Acarus siro $\mathrm{L}$. & $\begin{array}{l}\text { Chicken-pigeon-Turkey Hen- } \\
\text { Ducks }\end{array}$ & +++ & $\begin{array}{l}\text { Tanta-Qotor-Sakha- } \\
\text { Nasr city }\end{array}$ \\
\hline & Acarus farris Oudemans & $\begin{array}{c}\text { Chicken -Ducks-PekeenyDucks- } \\
\text { Geese- Turkey Hen }\end{array}$ & +++ & Tanta-Qotor-Sakha \\
\hline & Acarus gracillis Hughes & $\begin{array}{c}\text { Chicken-Pucks-Pigeon- Turkey } \\
\text { hen }\end{array}$ & ++ & $\begin{array}{l}\text { Tanta-Qotor-Sakha- } \\
\text { Nasr city }\end{array}$ \\
\hline & $\begin{array}{c}\begin{array}{c}\text { Tyrophagus neiswandri } \\
\text { Johnston\&Bruce }\end{array} \\
\text { Tyroborus } \mathrm{sp} . \\
\end{array}$ & Chicken & $\begin{array}{l}++ \\
+++ \\
\end{array}$ & Tanta \\
\hline & Caloglyphus rizoglyphoides & Poultry food & ++++ & Tanta-qotor -Sakha \\
\hline Chrotoglyphidae (1) & Chortoglyphus sp. & $\begin{array}{c}\text { Chicken -Ducks-Turkey Hen- } \\
\text { Pigeon-Quails }\end{array}$ & ++ & Tanta-Qotor \\
\hline Suidasiidae (1) & Suidasiones bitti (Koch) & All poultry & ++ & Sakha-Tanta \\
\hline \multirow{2}{*}{ Glycyphagidae (2) } & Ctenoglyphus plumiser (Koch) & Chicken & ++ & Tanta \\
\hline & Lepidoglyphus destructor (Schrank) & Chicken - Ducks & ++ & Sakha \\
\hline \multicolumn{5}{|c|}{ Suborder: Oribatida } \\
\hline Oribatalidae(1) & Zygoribatula tritici (El-Badry Nasr) & Chicken -Ducks-Turkey hen & + & Qotor-Sakha \\
\hline Oppidae(1) & Oppia sp. & $\begin{array}{c}\text { Ducks-Pekeeny-Ducks-Turkey } \\
\text { hen }\end{array}$ & ++ & Tanta \\
\hline
\end{tabular}

\section{REFERENCES}

Abo-Taka, S. M.1990. Mites inhabiting poultry farms in Egypt. In: Acarology 9, I proceedings. Ohio Bological Survey Columbus, Ohio: 97-990.
Abo-Taka, S. M. and Allam, S. A. 1997. Incidence, distribution, and ecology of ectoparasitic Acari infesting poultry farms in Menoufia Governorate, Egypt. Menofiya J. Agric. Res., 22 (2): 389-403. 
Griffiths, D. A. 1960. Some field habitats of mites of stored food products. Ann. Appl. Biol., 48(1): 134-144.

Hughes, A.M. 1961. The mites of stored food. Min. of Agr., Fish. \& Food Tech. Bull., 9:278.

Hughes, A. M. 1976. The mites of stored food products and houses. Tech. Bull., Min. Agric. and Fisheries in London, 9: $400 \mathrm{pp}$.

Karg, W. 1971. Acari (Acarina), Milben. Unterordung Anactinochaeta (Parasitiformes). Die freilebenden Gamasina (Gamasides), Raubmilben. Die Tierwelt Deutschlands, $59: 1-475$.

Krantz, G.W. and Walter, D. E. 2009. A Manual of Acarology.Texas Tech Univ. Press, 807 pp.

Lindquist, E. E. and Evans, G. O. 1965. Taxonomic concepts in the Ascidae, with a modified setal nomenclature for the idiosoma of the Gamasina (Acarina: Mesostigmata). Mem. Entomol. Soc. Can., 47: 1-64.

Mahgoob, A. E. A.; Tharwat, M. E.; Kilany, S. O.; Hafez, T. S. 2006. Mite fauna associated with some domestic and wild agricultural animals and their habitat in Egypt. Arab Universities Journal of Agricultural Sciences, 14(1):475-490.

Summers, F. M. and Price D.W., 1970. Review of the mite family Cheyletidae. Univ. Calif. Publ. Entomol., 61: 153 p.

Zaher, M.A. 1986. Survey and ecological studies on phytophagous, predacious and soil mites in Egypt II-A: Predacious and nonphytophagous mites PL. 480 Program U.S.A. Project No.EG-ARS-30 Grant No. FG 139: 518-567. 